\title{
Seric chemokines and chemokine receptors in eosinophils during acute human schistosomiasis mansoni
}

\author{
Denise Silveira-Lemos ${ }^{1,2,3,4}$, Andréa Teixeira-Carvalho²/+, Olindo Assis Martins-Filho², \\ Adriano Luiz Souza-Soares ${ }^{3}$, Pollyanna Castro-Silva1, Matheus Fernandes Costa-Silva, ${ }^{1,2}$, \\ Pedro Henrique Gazzinelli Guimarães ${ }^{1}$, Helena Barbosa Ferraz ${ }^{2}$, Lúcia Alves Oliveira-Fraga ${ }^{5}$, \\ Mauro Martins Teixeira ${ }^{3}$, Rodrigo Corrêa-Oliveira ${ }^{1}$
}

\begin{abstract}
${ }^{1}$ Laboratório de Imunologia Celular e Molecular ${ }^{2}$ Laboratório de Biomarcadores de Diagnóstico e Monitoração ${ }^{3}$ Departamento de Bioquímica e Imunologia, Instituto de Ciências Biológicas, Universidade Federal de Minas Gerais, Belo Horizonte, MG, Brasil ${ }^{4}$ Núcleo de Pesquisas em Ciências Biológicas, Laboratório de Imunoparasitologia, Departamento de Ciências Biológicas, Instituto de Ciências Exatas e Biológicas, Universidade Federal de Ouro Preto, Ouro Preto, MG, Brasil ${ }^{5}$ Núcleo de Pesquisa em Imunologia, Faculdade de Ciências da Saúde, Universidade Vale do Rio Doce, Governador Valadares, MG, Brasil
\end{abstract}

The recruitment of circulating eosinophils by chemokines and chemokine receptors plays an important role in the inflammation process in acute human schistosomiasis. Our main focus has been on the plasma chemokines (CXCL8/ CCL2/CCL3/CCL24) and chemokine receptors (CCR2/CCR3/CCR5/CXCR1/CXCR2/CXCR3/CXCR4) expressed by circulating eosinophils from acute Schistosoma mansoni infected patients (ACT). Our studies compared ACT patients and healthy individuals as a control group. Our major findings demonstrated a plethora of chemokine secretion with significantly increased secretion of all chemokines analysed in the ACT group. Although no differences were detected for beta-chemokine receptors (CCR2, CCR3 and CCR5) or alpha-chemokine receptors (CXCR3 and CXCR4), a significantly lower frequency of $C X C R 1^{+}$and $C X C R 2^{+}$eosinophils in the ACT group was observed. The association between chemokines and their chemokine receptors revealed that acutely infected schistosome patients displaying decreased plasma levels of CCL24 are the same patients who presented enhanced secretion of CCL3, as well as increased expression of both the CCR5 and CXCR3 chemokine receptors. These findings suggest that CCL24 may influence the kinetics of chemokines and their receptors and eosinophils recruitment during human acute schistosomiasis mansoni.

Key words: eosinophils - chemokine receptors - chemokines - acute schistosomiasis

Chemokines are a group of chemoattractant proteins that induce not only chemotaxis but also activation of target cells (Ulfman et al. 2001). It has been shown that some chemokines influence inflammatory cell infiltration and the cellularity that occurs in the granulomatous response around Schistosoma mansoni eggs. For instance, the chemokines macrophage inflammatory protein-1 $\alpha$ (MIP$1 \alpha /$ CCL3) and regulated upon activation normal T-cell expressed and secreted (RANTES/CCL5) are produced in a model of granulomatous inflammation in mice and ablation of their activity modulates the size of the lesions (Lukacs et al. 1993, 1994, Chensue et al. 1999, Qiu et al. 2001, Souza et al. 2005). Plasma levels of CCL3 are elevated in chronic schistosomiasis patients and correlate with the level of morbidity (Falcão et al. 2002, Souza et al. 2005); blocking CCL3 activity inhibits the granulomatous response in vitro (Falcão et al. 2002). Booth et al. (2004) recently reported that periportal fibrosis in males with $S$. mansoni infection is associated with low concentrations of CCL5.

Financial support: UNDP World Bank/WHO SPRTTD, CNPq (478320/2007-8), FAPEMIG (ATC, OAMF, MMT and RCO are CNPq fellowships)

+ Corresponding author: andreat@cpqrr.fiocruz.br

Received 6 January 2009

Accepted 16 October 2009
Eosinophils represent an important line of defence against parasitic infections (Butterworth 1984). In the parasitic disease caused by exposure to $S$. mansoni larvae, blood eosinophilia rises due to an increase in interleukin (IL)-5, IL-1, IL-3, granulocyte-macrophage colony-stimulating factor and granulocyte colony-stimulating factor (Clutterbuck et al. 1989, Weller 1992) and this increase is dependent on antigens secreted by eggs through the pores of the shell (Race et al. 1969, Sher et al. 1990). It is well established that an important step in the extravasation of eosinophils is their adhesion to the vascular endothelium, which is mediated by selectins and chemokines (Lampinen et al. 2004). However, the acute eosinophil cellular response against eggs and its contribution to granuloma formation is not well studied (Pacheco \& Lenzi 1997). Furthermore, there are no reports in the literature showing alteration in the plasma concentration of chemokines in acutely infected patients. Most of the current knowledge on the role of chemokines in schistosomiasis is derived from mice data. In contrast to experimentation in mice, there is a major difficulty faced by those researchers trying to answer questions in acute schistosomiasis in humans. People living in schistosome endemic areas, where it is easy to find infected patients, are unlikely to develop signs and symptoms related to the acute phase of schistosomiasis (Malhotra et al. 1997, King et al. 1998).

In the present study, a group of individuals who have not previously acquired schistosomiasis presented various characterised clinical symptoms of the early phase of 
S. mansoni infection. This allowed observation of certain immunological alterations in a cohesive group of acutely infected patients. In order to get a better insight into eosinophil participation in acute $S$. mansoni infection, we analysed the expression of chemokine receptors, namely, CCR2, CCR3, CCR5, CXCR1, CXCR2, CXCR3 and CXCR4 in the blood eosinophils of acutely infected patients and non-infected individuals. We also measured the levels of four different chemokines, CCL2 (MCP-1), CCL3 (MIP-1 $\alpha$ ), CCL24 (eotaxin-2) and CXCL8 (IL-8), which span different cellular specificities, in the plasma of these acute patients. Our findings led us to postulate that CCL24 may influence the kinetics of chemokines and their receptors and leukocyte recruitment during acute human schistosomiasis mansoni.

\section{PATIENTS, MATERIALS AND METHODS}

The study population consisted of two groups of individuals, acute $S$. mansoni-infected patients (ACT) and healthy blood donors as a control group (CT). The ACT group consisted of 10 patients (6 males and 4 females), with ages ranging from 14-21 years, who acquired their infection in the county village of São Geraldo da Piedade, an endemic area for $S$. mansoni infection, situated next to the city of Governador Valadares in the state of Minas Gerais (MG), Brazil. In our work, acute infected patients presented various characterized clinical symptoms associated with early infection by $S$. mansoni, such as fever, diarrhoea, headache, nausea, eosinophilia and, after a few weeks, the presence of schistosome eggs in their faeces. Quantitative parasitological stool examinations and detection of S. mansoni eggs was performed using Kato-Katz (Katz et al. 1972). The ultrasonography analysis did not show echogenicity abnormalities. ACT patients did not receive any corticosteroids or other immunosuppressant chemotherapy, nor were they treated for S. mansoni infection before blood collection. Upon positive laboratory diagnosis of schistosomiasis, all patients received treatment with a single dose of praziquantel, which was independent of their participation in this study.

Twenty-seven non-infected individuals (11 males and 16 females) were included as a CT group consisting of volunteer blood donors, aged from 19-30 years and were contacted at the Hemominas Blood Bank Foundation in Belo Horizonte, MG, Brazil. All non-infected volunteers were included after conclusive negative parasitological diagnosis of $S$. mansoni infection, in addition to negative serology for Chagas disease, leishmaniasis, human immunodeficiency virus infection and hepatitis.

The inclusion of all individuals in our investigation was preceded by their written agreement to participate, signed by the patient or by the patient's parents. This study was approved by the Ethical Committees of the Fundação Oswaldo Cruz-Fiocruz, Ministry of Health, Brazil.

White blood cell counts - Hemograms were performed in an automated haematology instrument (Coulter MD18, USA), using whole blood collected in $5 \mathrm{~mL}$ vacutainer tubes containing ethylenediamine tetraacetic acid (EDTA) as the anticoagulant (Becton Dickinson Biosciences, San Diego, CA, USA). The parameter measured was a differential analysis of leukocyte subsets, including absolute counts of eosinophils, neutrophils, lymphocytes and monocytes.
Quantification of plasma chemokines by ELISA - The plasma concentrations of chemokines were measured by sandwich enzyme-linked immunosorbent assay (ELISA), as described by Morita et al. (1999). The ELISA kits for human CCL2 and CCL24 were obtained from R\&D Systems, Minneapolis, MN, USA. The antibody pairs and chemokines standards used in the assay for human CCL3 and CXCL8 were purchased from Pharmingen (San Diego, CA, USA). The specific recommendations of the manufacturers were followed. Human plasma samples were subjected to acid-salt precipitation to avoid cross-reactivity in ELISA (Falcão et al. 2002). The concentration of chemokines in plasma was analysed in $\mathrm{pg} / \mathrm{mL}$.

Immunophenotypicanalysis byflowcytometry-Human monoclonal antibodies (mAbs) for anti-CCR2 (48607.211), anti-CCR3 (61828.111), anti-CCR5 (45502.111), anti-CXCR1 (42705.111), anti-CXCR2 (48311.211), anti-CXCR3 (49801.111) and anti-CXCR4 (12G5) were labelled with fluorescein isothiocyanate (FITC) (R\&D systems). Antimouse IgG1 (679.1Mc7) was used as isotype control and purchased from Becton Dickinson Biosciences Pharmingen (San Diego, CA, USA).

Cell staining procedure - One hundred microlitres of EDTA whole blood was incubated in the presence of 5 $\mu \mathrm{L}$ of undiluted anti-human cell surface molecules mAbs for $30 \mathrm{~min}$ in the dark and at room temperature (RT). For CCR2 analysis, monoclonal antibody labelled with biotin (R\&D systems, USA) was diluted $1 / 5$ in phosphatebuffered saline (PBS) with avidin-FITC (Diatec, Oslo, Norway) diluted $1 / 100$ in PBS. In this analysis, the cells were incubated with $50 \mu \mathrm{L}$ of diluted monoclonal antibody in the dark for $30 \mathrm{~min}$ at RT. Then, erythrocyte lysis was performed using $2 \mathrm{~mL}$ of FACS Lysing Solution (Becton Dickinson Biosciences Pharmingen, San Diego, CA, USA), followed by incubation for $10 \mathrm{~min}$ at room temperature (RT). The leukocyte suspension was further washed with $2 \mathrm{~mL}$ of PBS containing $0.01 \%$ sodium azide. Prior to flow cytometric acquisition, stained cells were fixed in $200 \mu \mathrm{L}$ of FACS fix solution $(10 \mathrm{~g} / \mathrm{L}$ paraformaldehyde, $10.2 \mathrm{~g} / \mathrm{L}$ sodium cacodylate, $6.65 \mathrm{~g} / \mathrm{L}$ sodium chloride) for at least $15 \mathrm{~min}$ at $4^{\circ} \mathrm{C}$. Data collection from 20,000 events was performed using a FACScan flow cytometer (Becton Dickinson, Mountain View, CA, USA). CELLQUEST ${ }^{\mathrm{TM}}$ software provided by the manufacturer was used for data acquisition and analysis.

Analysis of eosinophils was performed by single colour immunophenotyping with FL-1/FITC-labelled anti-cell surface marker mAbs. The gating strategy was based on eosinophil selection by their autofluorescent properties in a non-related FL-3 channel vs. forward scatter (FSC) graphs, as described by Weil and Chused (1981). Eosinophil subpopulations were identified using FSC vs. FL3 dot plots. A confirmatory graph was also set up to identify the eosinophils on FSC vs. side scatter dot plot distributions confined to a region of high cell complexity. Data are presented as the percentage of eosinophils expressing a given phenotypic marker, except for CCR3, which was subjected to a semi-quantitative analysis of a single colour histogram of FL1/FITC fluorescence intensity performed as a fluorescence mean channel. 
Statistics - Differences between groups were first evaluated by MINITAB 13.20 software (San Diego, CA, USA) to examine three parameters, independence, normality and variance, of the data sets. Considering their non-parametric nature, all data sets were analysed using Mann-Whitney's test, with further analysis by Chi-square and Spearman's rank correlation tests using the Graphpad PRISM 4.02 software (La Jolla, CA, USA). Significance was defined in all cases at $\mathrm{p}<0.05$.

\section{RESULTS}

Acute human schistosomiasis is accompanied by eosinophilia and monocytosis - The assessment of hematological parameters was evaluated in Fig. 1. Data analysis demonstrated that the ACT group displayed a significant increase in the absolute counts of eosinophils as compared to the CT group (Fig. 1A). This eosinophilic profile was accompanied by a significant increase in monocytes $(\mathrm{p}=$ $0.003)$ besides of a decrease in the neutrophils $(p=0.0373)$ counts in the ACT group as compared to the CT group (Fig. 1C, D, respectively). No significant differences were observed for lymphocyte absolute values (Fig. 1B).
Increased plasma levels of alpha/beta-chemokines are found during acute human schistosomiasis mansoni - The ex vivo analysis of the alpha and beta-chemokine patterns in acute patients is illustrated in Fig. 2. Interestingly, data analysis demonstrated a plethora of chemokines secretion with a significant increase in secretion of all chemokines analysed [CCL2 (Fig. 2A), CCL3 (Fig. 2B), CCL24 (Fig. 2C) and CXCL8 (Fig. 2D)] in the ACT group as compared to the CT group. In order to extend our initial findings, we performed a detailed correlation analysis of circulating eosinophil subset counts and chemokine levels. The results of this investigation revealed a negative correlation between the levels of CCL2 and the number of eosinophils $(r=-0.64 ; p<0.05)$ (data not shown). No additional significant correlations were found with the additional chemokines investigated.

Diminished expression of the alpha-chemokine receptors CXCR1 and CXCR2 is observed during acute human schistosomiasis mansoni - The pattern of alpha and beta-chemokine receptor expression on eosinophils during acute schistosomiasis mansoni is illustrated in
A

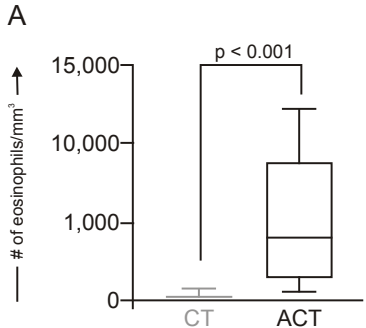

B

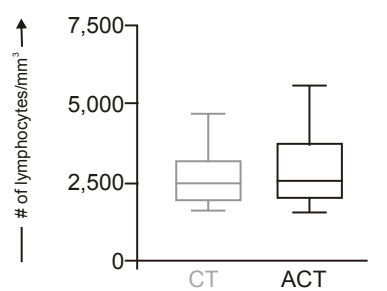

C

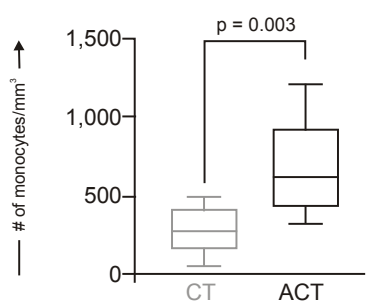

D

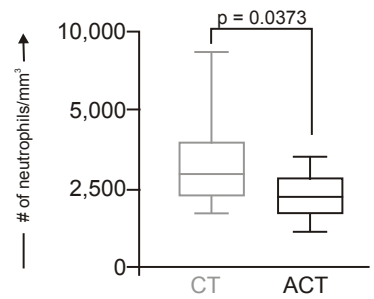

Fig. 1: analysis of leukocytes subsets (A: eosinophils; B: lymphocytes; C: monocytes; D: neutrophils) in the peripheral blood of acute patients (ACT, $\square=10$ ) or control group (CT, $\square=27$ ). The results are expressed in box-plot format. The box stretches from the lower hinge (defined as the 25 th percentile) to the upper hinge (the 75th percentile) and therefore contains the middle half of the scores in the distribution. The median is shown as a line across the box. Therefore $1 / 4$ of the distribution is between this line and the top of the box and $1 / 4$ of the distribution is between this line and the bottom of the box. Significant differences (connecting lines) were considered at $\mathrm{p}<0.05$.
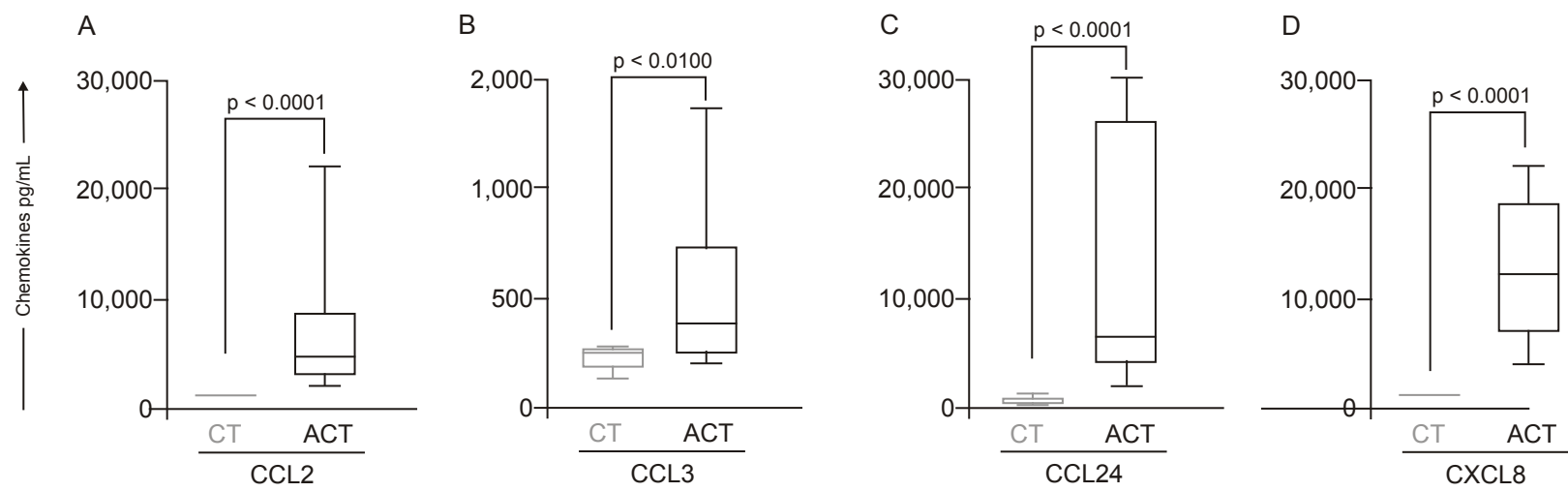

Fig. 2: plasmatic levels of alpha and beta-chemokines from acute Schistosoma mansoni infected patients (ACT, $\square=10$ ) and control group $(\mathrm{CT}, \square=27$ ). The results are expressed in box-plot format highlighting the gap of $50 \%$ of data set measurement for the CCL2 (A), CCL3 (B), CCL24 (C) and CXCL8 (D). Significant differences (connecting lines) were considered at $\mathrm{p}<0.05$. 
Fig. 3. No differences were detected for beta-chemokine receptors, including the percentage of $\mathrm{CCR} 2^{+}$or $\mathrm{CCR} 5^{+}$ eosinophils and CCR3 eosinophil expression (Fig. 3A$\mathrm{C}$, respectively) as well as for the alpha-chemokine receptors CXCR3 and CXCR4 (Fig. 3F, G, respectively). Interestingly, data analysis demonstrated a shift in the alpha-chemokine receptor profile characterised by a significantly lower frequency of CXCR $1^{+}$and CXCR2 ${ }^{+}$ eosinophils ( $p=0.0458$ and $p=0.0084$, respectively) in the ACT group as compared to the CT group (Fig. 3D, E, respectively). In addition, a positive correlation between CCR2 and CCR5 expression and eosinophil absolute counts was observed $(\mathrm{r}=0.66, \mathrm{p}<0.05 ; \mathrm{r}=0.74, \mathrm{p}<$ 0.05 , respectively) (data not shown).

Decreased plasma levels of CCL24 are associated with enhanced secretion of CCL3 and increased expression of CCR5 and CXCR3 in eosinophil subsets during acute $S$. mansoni infection - The patterns of plasma levels of chemokines and chemokine receptor expression are shown in Fig. 4. Data analysis revealed that ACT patients who displayed decreased plasma levels of CCL24 are the same patients who presented enhanced secretion of CCL3 (Fig. 4A) ( $p<0.0001)$, as well as increased expression of both CCR5 and CXCR3 chemokine receptors (Fig. 4B) $(p=0.002$ and $p=0.0337$, respectively). In addition, we performed a further investigation that revealed a direct association between CCL3 levels and the percentage of
$\mathrm{CCR}^{+}$or $\mathrm{CXCR}^{+}$eosinophils (Fig. $\left.4 \mathrm{C}\right)(\mathrm{p}=0.0001)$ during acute human schistosomiasis mansoni.

In order to confirm the initial findings, we performed a correlation between levels of CCL24 and percentage of $\mathrm{CCR}^{+}$or $\mathrm{CXCR}^{+}$eosinophils (Fig. 4D). The results demonstrated the existence of a strong negative correlation between levels of CCL24 and the frequency of CCR 5 or CXCR3 in eosinophils from acute patients ( $\mathrm{r}$ $=-0.7833, \mathrm{p}=0.001 ; \mathrm{r}=-0.7333, \mathrm{p}=0.001$, respectively). Together, these findings led us to postulate that CCL24 may influence the kinetics of chemokines and their receptors, as well as leukocyte recruitment during human acute schistosomiasis mansoni.

\section{DISCUSSION}

Eosinophilia is a hallmark of the acute phase of schistosomiasis - Eosinophils represent a minor cell type among leukocytes, with basal counts ranging from 400600 cells $/ \mathrm{mm}^{3}$ (Brito-Babapulle 2003). In this study, acute schistosomiasis patients had a significantly higher number of eosinophils. Experimental studies have demonstrated that eosinophilia occurs between the 5th-7th weeks after exposure to the parasite and may be induced by Th2 cytokines, such as IL-4, IL-10, IL-13, IL-9 and especially IL-5 (Cara et al. 2000). Hoshi et al. (1995) demonstrated that a transient arrest of rolling eosinophils may be induced by chemokine CXCL 8 and shifted into firm long-term arrest by CCL24. Eosinophils then

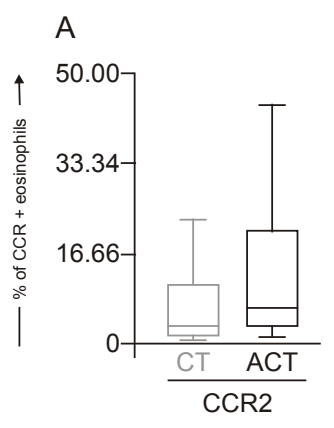

\section{B}

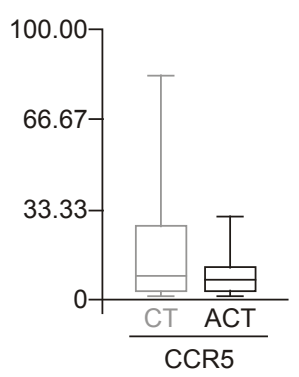

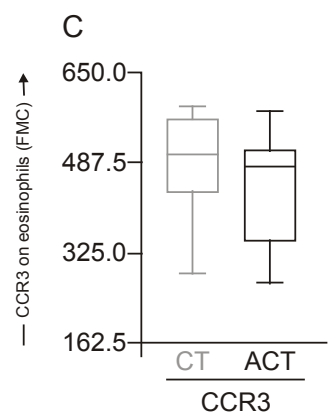

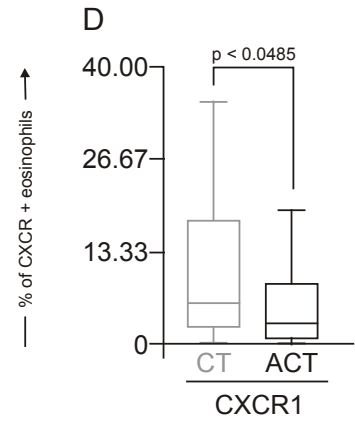

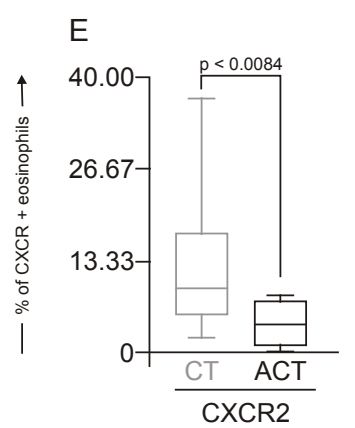
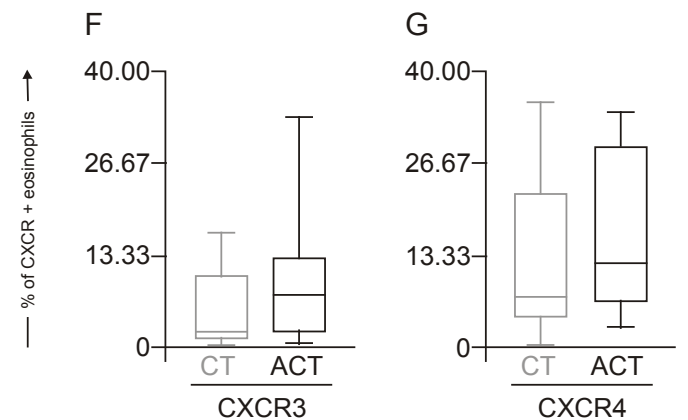

Fig. 3: alpha and beta-chemokine receptor expression by peripheral blood eosinophils from Schistosoma mansoni-infected patients (ACT, $\square=10$ ) and control group (CT, $\square=27$ ). The selection of eosinophils was essentially based on their autofluorescent cells using non-related FL-3 channel versus forward scatter (FSC) and the analysis of chemokine receptor ${ }^{+}$eosinophils was carry out by single color immunophenotyping. The results are expressed in box-plot format highlighting the gap of 50\% of data set measurement for the percentage of CCR2 (A), CCR5 (B), CXCR1 (D), CXCR2 (E), CXCR3 (F), CXCR4 (G) cells and the fluorescence mean channel (FMC) of CCR3 expression by eosinophils (C). Significant differences (connecting lines) were considered at $\mathrm{p}<0.05$. 


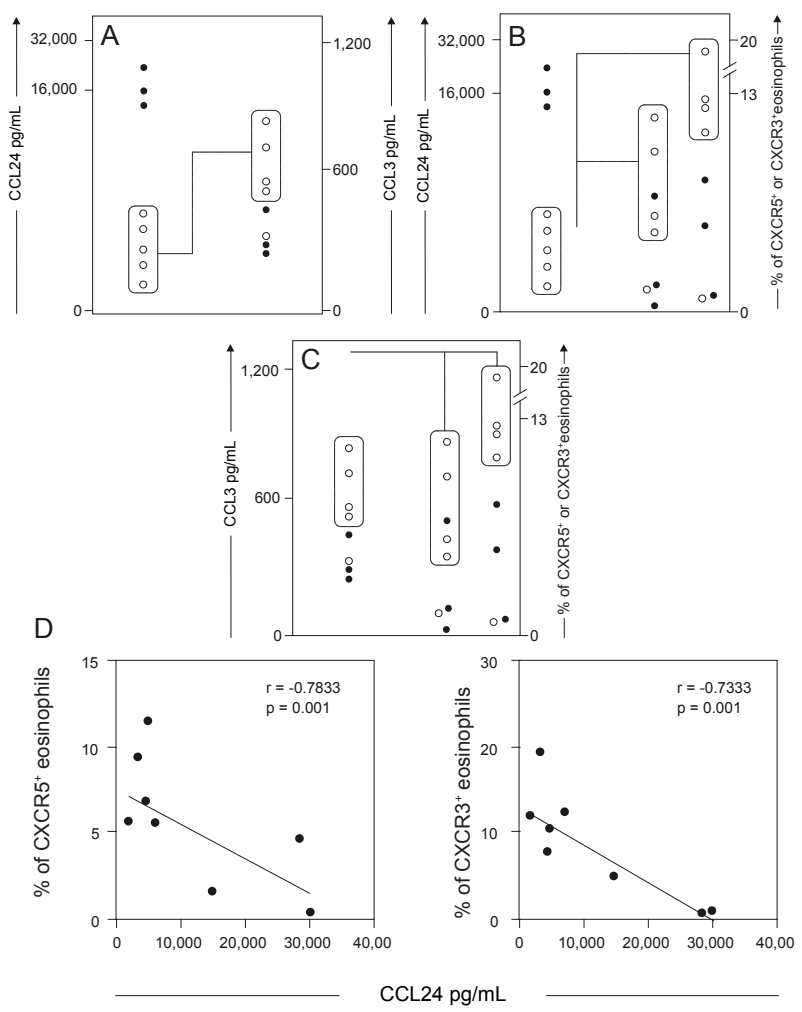

Fig. 4: analysis of major discriminatory immunophenotypes of Schistosoma mansoni-infected patients $(\mathrm{ACT}=8)$. A dichotomic pattern of CCL24 levels divides the individuals in two subgroups: high producers $(\bullet)$ and low producers $(\bigcirc)$. Analysis of individual data from ACT demonstrates an association (dotted rectangles and lines) between low levels of CCL24 with high levels of CCL3 (A), high frequency of $\mathrm{CCR}^{+}$and high frequency of CXCR $3^{+}$eosinophils (B). Analysis of individual data from ACT patients demonstrates an association (dotted rectangles and lines) between the high levels of CCL3 with high frequency of both $\mathrm{CCR}^{+}$and $\mathrm{CXCR}^{+}$eosinophils (C). Confirmatory correlation analysis validates the negative association between levels of CCL24 and frequency of CCR $5^{+}$or $\mathrm{CXCR}^{+}$eosinophils (D). Correlation analyses ( $r$ and $p$-values) are shown in the figure (D). Significant differences were considered at $\mathrm{p}<0.05$.

migrate into the tissue in response to chemotactic factors produced locally at the inflammatory site (Teran et al. 1996). Smithers et al. (1977) demonstrated in experimental models that the cellular infiltrate observed in hepatic granulomas is predominantly formed by eosinophils and monocytes.

Chemokines in the plasma of acute schistosomiasis patients - The present paper represents the first report of increased chemokine concentrations in the plasma of S. mansoni acute-infected patients. All four of the chemokines measured, CCL2, CCL3, CCL24 and CXCL8, were found at higher levels in the plasma of schistosomiasis patients in the acute phase of the disease as compared to healthy individuals. Although the presence of these chemokines in plasma is not enough to show a definitive role in the manifestations and general immune response associated with acute schistosomiasis in humans, some evidence is available from animal models.
CCL24 and CXCL8 are generally known for their chemotactic activity towards eosinophils and neutrophils, respectively. CCL24 is selectively agonistic towards CCR3 receptors, whose expression on eosinophils (Heath et al. 1997), basophils (Uguccioni et al. 1997) and Th2 lymphocytes (Gerber et al. 1997, Sallusto et al. 1998) is compatible with a role in allergic disease and the immune response against helminth infections. In this context, CCL24 could attract eosinophils to inflammatory sites to participate in the initial formation of granulomas. This cell type may comprise up to $40 \%$ of the cellular composition of granulomas. Moreover, an increase of CCL24 could indicate a Th2 type immune response. The role of CXCL8 is more evident in acute inflammation, where this chemokine attracts neutrophils to inflammatory sites (Broaddus et al. 1994). Although neutrophils are not obvious contributors to the formation of schistosome granulomatous, this cell type may have a role in the initial influx of cells or at the interface between innate and acquired immunity, collaborating in the secretion of inflammatory mediators that will bring other cells types of greater importance. A decreased frequency of circulating neutrophils in acute schistosomiasis patients may indicate a migration of this cell type into the inflammatory focus. This seems plausible since blood cells from infected patients release mediators in response to soluble eggs antigen stimulation that are chemotactic to neutrophils and eosinophils (Dabes et al. 1989). Moreover, schistosome eggs themselves secrete antigens that have direct chemotactic activity on neutrophils and eosinophils (Owhashi et al. 1985).

The chemokines CCL2 and CCL3 are important mediators of the influx of monocytes and macrophages, which are relevant cellular types in the granulomatous response around schistosome eggs. In addition, both macrophages (Koch et al. 1992) and eosinophils (Izumi et al. 1997) are sources of CCL2. Tillie-Leblond et al. (2000) demonstrated that chemotactic activity from eosinophils from bronchial lavage fluid in asthmatic subjects is associated with higher levels of CCL2, CCL3 and CCL5. Moreover, CCL3-deficient schistosome-infected mice exhibit smaller granulomas compared to wild-type mice (Souza et al. 2005). In humans, a correlation between high levels of CCL3 and severity of disease has been observed during schistosome infection (Falcão et al. 2002). Thus, CCL2 and CCL3 could promote the accumulation of macrophages and eosinophils in the early phases of granuloma formation. In our study, both chemokines are increased in plasma from patients with acute $S$. mansoni infection.

Expression profile of chemokines receptors in eosinophils - Signals via chemokine receptors play an important role in the accumulation of eosinophils (Nagase et al. 2001). Previous data have demonstrated a constitutive and as well as or inducible expression of CCR1, CCR3, CXCR1, CXCR2, CXCR3 and CXCR4 by human circulating eosinophils (Heath et al. 1997). In our study, we observed a decrease in the expression of both CXCR1 and CXCR2 by eosinophils from acute patients compared to healthy individuals and a positive correlation between the expression of CCR2 and CCR5 in the ACT group. 
Schuh et al. (2002), while studying CXCR2 knockout mice during allergic airway reactions and asthma, demonstrated that this chemokine receptor may have significant role in the development and maintenance of both diseases. Heath et al. (1997) demonstrated that after 5-7 days culture in vitro with human IL-5, CXCR2 and to a lesser degree CXCR1 were detectable on the surface of eosinophils and this expression is in parallel with the ability of these eosinophils to migrate to CXCL8 in chemotaxis assays. However, there are conflicting reports on the expression of CXCR2 on circulating eosinophils (Petering et al. 1999, Jinquan et al. 2000, Nagase et al. 2001) and little is known about the expression of chemokine receptors on peripheral blood eosinophils in patients with acute schistosomiasis. Our data suggest that CXCR1 and CXCR2 are not necessary for the activation of circulating eosinophils in the ACT group.

Influence of CCL24 on chemokines and their receptors - The study of the importance of chemokines and their receptors for development and maintenance of acute human schistosomiasis has been neglected. Of particular interest are our data describing novel associations between CCL24 and chemokines, such as CCL3, as well as chemokine receptors such as CCR5 and CXCR3. Eotaxin-2 (CCL24) has long been known to be a specific agonist for CCR3, attracting and activating eosinophils, basophils and Th2 type T lymphocytes and its expression profile coincides with a potential role in allergic inflammation or parasitic diseases as schistosomiasis (Petkovic et al. 2004, Silveira-Lemos et al. 2008). Recently, it was reported that eotaxin-3 (CCL26) acts as a natural antagonist on CCR1, CCR2 and CCR5 receptors (Ogilvie et al. 2003, Petkovic et al. 2004) and may play an unrecognised role in the polarisation of cellular recruitment by attracting Th2 lymphocytes, eosinophils and basophils via CCR3, while concomitantly blocking the recruitment of Th1 lymphocytes and monocytes via CCR1, CCR2 and CCR5. Our data showed a strong negative correlation between levels of CCL24 and expression of CCR5 and CXCR3, suggesting that a similar mechanism may be occurring during acute schistosomiasis. In this context, the increased responsiveness of eosinophils from acute patients to CCL3 may be mediated by their specific receptors, such as CCR5. Oliveira et al. (2002) investigated the regulation of chemokine-mediated responses and receptor expression on eosinophils from mice and reported that CCL3 and eotaxin-1 (CCL11) induced a significant intracellular calcium flux in antigenelicited and peripheral blood eosinophils and that CCL2, CCL22, CCL4 and CCL1 did not. Peripheral blood eosinophils migrated toward CCL3 and CCL11 but did not migrate toward CCL2, CCL22, CCL4 or CCL1. These results suggest that eosinophils may up-regulate and use additional chemokine receptors during the progression of inflammatory and stimulus responses for migration and activation and that the chemokine milieu is very important in this process.

Overall, our data demonstrated that the acute phase of schistosomiasis is characterised by an eosinophilic profile that shows the complex relationship between chemokines and their receptors and that it may affect the distribution and recruitment of immune cells towards an inflammatory focus as well as determine the establishment of distinct patterns of immunological parameters during human acute schistosomiasis mansoni.

\section{ACKNOWLEDGEMENTS}

To the technical staff of the Laboratorio de Imunologia Celular e Molecular, Fundação Oswaldo Cruz-Fiocruz, and the Núcleo de Pesquisa em Imunologia, UNIVALE, Brazil, for invaluable assistance during this study, and to the PDTISFiocruz, for use of its facilities.

\section{REFERENCES}

Booth M, Mwatha JK, Joseph S, Jones FM, Kadzo H, Ireri E, Kazibwe F, Kemijumbi J, Kariuki C, Kimani G, Ouma JH, Kabatereine NB, Vennervald BJ, Dunne DW 2004. Periportal fibrosis in human Schistosoma mansoni infection is associated with low IL-10, low IFN-gamma, high TNF-alpha, or low RANTES, depending on age and gender. J Immunol 15: 1295-1303.

Brito-Babapulle F 2003. The eosinophilias including the idiopathic hypereosinophilic syndrome. Br J Haematol 121: 203-223.

Broaddus VC, Boylan AM, Hoeffel JM, Kim KJ, Sadick M, Chuntharapai A, Hébert CA 1994. Neutralization of IL-8 inhibits neutrophil influx in a rabbit model of endotoxin-induced pleurisy. J Immunol 15: 2960-2967.

Butterworth AE 1984. Cell-mediated damage to helminths. Adv Parasitol 23: 143-235.

Cara DC, Negrao-Correa D, Teixeira MM 2000. Mechanisms underlying eosinophil trafficking and their relevance in vivo. Histol Histopathol 15: 899-920.

Chensue SW, Warmington KS, Allenspach EJ, Lu B, Gerard C, Kunkel SL, Lukacs NW 1999. Differential expression and cross-regulatory function of RANTES during mycobacterial (type 1) and schistosomal (type 2) antigen-elicited granulomatous inflammation. J Immunol 163: 165-173.

Clutterbuck EJ, Hirst EM, Sanderson CJ 1989. Human interleukin-5 (IL-5) regulates the production of eosinophils in human bone marrow cultures: comparison and interaction with IL-1, IL-3, IL-6 and GMCSF. Blood 73: 1504-1512.

Dabes TM, Garcia AA, Colley DG, Ramalho-Pinto FJ 1989. Lymphokine production by blood or spleen mononuclear cells from patients with schistosomiasis mansoni. Am J Trop Med Hyg 40: 273-281.

Falcão PL, Correa-Oliveira R, Fraga LA, Talvani A, Proudfoot AE, Wells TN, Williams TJ, Jose PJ, Teixeira MM 2002. Plasma concentrations and role of macrophage inflammatory protein-1alpha during chronic Schistosoma mansoni infection in humans. J Infect Dis 186: 1696-1700.

Gerber BO, Zanni MP, Uguccioni M, Loetscher M, Mackay CR, Pichler WJ, Yawalkar N, Baggiolini M, Moser B 1997. Functional expression of the eotaxin receptor CCR3 in T lymphocytes colocalizing with eosinophils. Curr Biol 7: 836-843.

Heath H, Qin S, Rao P, Wu L, LaRosa G, Kassam N, Ponath PD, Mackay CR 1997. Chemokine receptor usage by human eosinophils. The importance of CCR3 demonstrated using an antagonistic monoclonal antibody. J Clin Invest 99: 178-184.

Hoshi H, Ohno I, Honma M, Tanno Y, Yamauchi K, Tamura G, Shirato K 1995. IL-5, IL-8 and GM-CSF immunostaining of sputum cells in bronchial asthma and chronic bronchitis. Clin Exp Allergy 25: 720-728. 
Izumi S, Hirai K, Miyamasu M, Takahashi Y, Misaki Y, Takaishi T, Morita Y, Matsushima K, Ida N, Nakamura H, Kasahara T, Ito K 1997. Expression and regulation of monocyte chemoattractant protein-1 by human eosinophils. Eur J Immunol 27: 816-824.

Jinquan T, Jing C, Jacobi HH, Reimert CM, Millner A, Quan S, Hansen JB, Dissing S, Malling HJ, Skov PS, Poulsen LK 2000. CXCR3 expression and activation of eosinophils: role of IFN-gamma-inducible protein-10 and monokine induced by IFN-gamma. J Immunol 165: 1548-1556.

Katz N, Chaves A, Pellegrino JP 1972. A simple device for quantitative thick-smear technique in schistosomiasis mansoni. Rev Inst Med Trop Sao Paulo 14: 397-400.

King CL, Malhotra I, Mungai P, Wamachi A, Kioko J, Ouma JH, Kazura JW 1998. B cell sensitization to helminthic infection develops in utero in humans. J Immunol 160: 3578-3584.

Koch AE, Kunkel SL, Harlow LA, Johnson B, Evanoff HL, Haines GK, Burdick MD, Pope RM, Strieter RM 1992. Enhanced production of monocyte chemoattractant protein-1 in rheumatoid arthritis. J Clin Invest 90: 772-779.

Lampinen M, Carlson M, Håkansson LD, Venge P 2004. Cytokineregulated accumulation of eosinophils in inflammatory disease. Allergy 59: 793-805.

Lukacs NW, Chensue SW, Smith RE, Strieter RM, Warmington K, Wilke C, Kunkel SL 1994. Production of monocyte chemoattractant protein-1 and macrophage inflammatory protein-1 alpha by inflammatory granuloma fibroblasts. Am J Pathol 144: 711-718.

Lukacs NW, Kunkel SL, Strieter RM, Warmington K, Chensue SW 1993. The role of macrophage inflammatory protein 1 alpha in Schistosoma mansoni egg-induced granulomatous inflammation. J Exp Med 177: 1551-1559.

Malhotra I, Ouma J, Wamachi A, Kioko J, Mungai P, Omollo A, Elson L, Koech D, Kazura JW, King CL 1997. In utero exposure to helminth and mycobacterial antigens generates cytokine responses similar to that observed in adults. $J$ Clin Invest 99: 1759-1766.

Morita A, Shimosako K, Kikuoka S, Taniguchi Y, Kitaura M, Sasakura K, Tamaki M, Tsuji T, Teraoka H, Yoshie O, Nakajima T, Hirai K 1999. Development of a sensitive enzyme-linked immunosorbent assay for eotaxin and measurement of its levels in human blood. J Immunol Methods 226: 159-167.

Nagase H, Kudo K, Izumi S, Ohta K, Kobayashi N, Yamaguchi M, Matsushima K, Morita Y, Yamamoto K, Hirai K 2001. Chemokine receptor expression profile of eosinophils at inflamed tissue sites: decreased CCR 3 and increased CXCR4 expression by lung eosinophils. J Allergy Clin Immunol 108: 563-569.

Ogilvie P, Paoletti S, Clark-Lewis I, Uguccioni M 2003. Eotaxin-3 is a natural antagonist for CCR2 and exerts a repulsive effect on human monocytes. Blood 102: 789-794.

Oliveira SH, Lira S, Martinez-A C, Wiekowski M, Sullivan L, Lukacs NW 2002. Increased responsiveness of murine eosinophils to MIP-1beta (CCL4) and TCA-3 (CCL1) is mediated by their specific receptors, CCR5 and CCR8. J Leukoc Biol 71: 1019-1025.

Owhashi M, Horii Y, Ishii A 1985. Schistosoma japonicum: identification and characterization of neutrophil chemotactic factors from egg antigen. Exp Parasitol 60: 229-238.

Pacheco RG, Lenzi HL 1997. Systemic modulation of peripheral eosinophilia (air pouch model) in Schistosoma mansoni infection. Mem Inst Oswaldo Cruz 92 (Suppl. II): 165-172.

Petering H, Götze O, Kimmig D, Smolarski R, Kapp A, Elsner J 1999. The biologic role of interleukin-8: functional analysis and expression of CXCR1 and CXCR2 on human eosinophils. Blood 93: 694-702.
Petkovic V, Moghini C, Paoletti S, Uguccioni M, Gerber B 2004. Eotaxin-3/CCL26 is a natural antagonist for CC chemokine receptors 1 and 5. A human chemokine with a regulatory role. J Biol Chem 279: 23357-23363.

Qiu B, Frait KA, Reich F, Komuniecki E, Chensue SW 2001. Chemokine expression dynamics in mycobacterial (type-1) and schistosomal (type-2) antigen-elicited pulmonary granuloma formation. Am J Pathol 158: 1503-1515.

Race GJ, Michaels RM, Martin JH, Larsh JEJr, Matthews JL 1969. Schistosoma mansoni eggs: an electron microscopic study of shell pores and microbarbs. Proc Soc Exp Biol Med 130: 990-992.

Sallusto F, Lanzavecchia A, Mackay CR 1998. Chemokines and chemokine receptors in T-cell priming and Th1/Th2-mediated responses. Immunol Today 19: 568-574.

Schuh JM, Blease K, Hogaboam CM 2002. CXCR2 is necessary for the development and persistence of chronic fungal asthma in mice. J Immunol 168: 1447-1456.

Sher A, Coffman RL, Hieny S, Scott P, Cheever AW 1990. Interleukin 5 is required for the blood and tissue eosinophilia but not granuloma formation induced by infection with Schistosoma mansoni. Proc Natl Acad Sci USA 87: 61-65.

Silveira-Lemos D, Teixeira-Carvalho A, Martins-Filho OA, Alves Oliveira LF, Costa-Silva MF, Matoso LF, de Sousa LJ, Gazzinelli A, Corrêa-Oliveira R 2008. Eosinophil activation status, cytokines and liver fibrosis in Schistosoma mansoni infected patients. Acta Trop 108: 150-159.

Smithers SR, McLaren DJ, Ramalho-Pinto FJ 1977. Immunity to schistosomes: the target. Am J Trop Med Hyg 26: 11-19.

Souza AL, Roffê E, Pinho V, Souza DG, Silva AF, Russo RC, Guabiraba R, Pereira CA, Carvalho FM, Barsante MM, Correa-Oliveira R, Fraga LA, Negrão-Correa D, Teixeira MM 2005. Potential role of the chemokine macrophage inflammatory protein 1 alpha in human and experimental schistosomiasis. Infect Immun 73: 2515-2523.

Teran LM, Noso N, Carroll M, Davies DE, Holgate S, Schröder JM 1996. Eosinophil recruitment following allergen challenge is associated with the release of the chemokine RANTES into asthmatic airways. J Immunol 157: 1806-1812.

Tillie-Leblond I, Hammad H, Desurmont S, Pugin J, Wallaert B, Tonnel AB, Gosset P 2000. CC chemokines and interleukin-5 in bronchial lavage fluid from patients with status asthmaticus. Potential implication in eosinophil recruitment. Am J Respir Crit Care Med 162: 586-592.

Uguccioni M, Mackay CR, Ochensberger B, Loetscher P, Rhis S, LaRosa GJ, Rao P, Ponath PD, Baggiolini M, Dahinden CA 1997. High expression of the chemokine receptor CCR3 in human blood basophils. Role in activation by eotaxin, MCP-4 and other chemokines. J Clin Invest 100: 1137-1143.

Ulfman LH, Joosten DP, van der Linden JA, Lammers JW, Zwaginga JJ, Koenderman L 2001. IL-8 induces a transient arrest of rolling eosinophils on human endothelial cells. J Immunol 166: 588-595.

Weil GJ, Chused TM 1981. Eosinophil autofluorescence and its use in isolation and analysis of human eosinophils using flow microfluorometry. Blood 57: 1099-1104.

Weller PF 1992. Cytokine regulation of eosinophil function. Clin Immunol Immunopathol 62: S55-S59. 